\title{
Safer Hybrid Workspace Using Human-Robot Interaction While Sharing Production Activities
}

\author{
Ramy Meziane, Ping Li, Martin J.-D.Otis, Hassan Ezzaidi \\ REPARTI Center, University of Quebec at Chicoutimi \\ Chicoutimi, Canada \\ Email: Martin_Otis@uqac.ca
}

\author{
Philippe Cardou \\ REPARTI Center, Laval University \\ Quebec, Canada \\ Email: pcardou@gmc.ulaval.ca
}

\begin{abstract}
In a near future, human and industrial manipulator will work together sharing a common workspace and production activities leading to a potential increase of accident. The research project concerns the adaptation of industrial robot already installed in a flexible manufacturing system in order to make it more interactive with human. The aim concerns the reduction of potential risk of injuries while working with an industrial robot. This paper presents a new inexpensive, non-intrusive, non-invasive, and non-vision-based system, for human detection and collision avoidance. One method investigated for improving safety concerns planning of safe path. This system recognizes human activities and locates operator's position in real time through an instrumented safety helmet. This safety helmet includes an IMU (Inertial Measurement Unit) and an indoor localization system such as RSSI (Received Signal Strength Indication) using industrial wireless equipment. A hybrid workspace including a flexible manufacturing system has been designed in order to practice experiments in an industrial-like environment.
\end{abstract}

Keywords - Activity recognition; collision avoidance; humanrobot interaction; Safety hybrid workspace; RSSI; IMU.

\section{INTRODUCTION}

Nowadays, in the industrial sector, new requirements involve flexibility and reactivity in production line in order to allow fast modification in product characteristics. Human and robot interactions seem more suited for allowing fast adjustment of production sequences. Moreover, introduction of human-robot interaction (HRI) in industrial sector, allow to reduce physical efforts and to preserve the operator's health. HRI then could improve production. Indeed, the mechanical properties of the robot manipulators complete those of human. Robot manipulators are particularly optimized to have strength, rigidity and precision abilities while human gives operational decisions in processes.

Several forms of HRI in a shared workspace could be found in the scientific literature. For example the robot can be used like weight compensation for heavy or bulky assembly parts. These robots are called COBOT «collaborative robot» and was introduced in 1999 by Edward Colgate and Michael Peshkin. Another example is the Intelligent Assist Device (IAD) system. They are a manipulator with lower degrees of freedom controlled by an operator using physical interactions. Both IAD and COBOT are different systems than exosqueleton since the operator is outside the device.

In contrast with IAD, COBOT system can detect and avoid collisions with human and environmental objects. COBOTs are mainly used to help the workers to lift, move production workloads and track assembly line. They can also place the loads quickly, precisely and safely. These systems are mainly employed in automotive industry [1]. More recently, they were used for sharing production activities which robot and human perform different tasks in the same workspace. Therefore, a new form of interaction appeared: working time sharing. Operator and robot are able to perform a joint task manipulation or handling [2]. This collaborative work within a common workspace is labelled hybrid workspace in the following. An overview on these forms of interactions and technologies used in assembly lines are presented in [3]. However, workspace sharing with the robot manipulator is still dangerous for human safety $[4,5]$ and this issue need further investigations.

Accidents with robots occurred during manipulations tasks and furthermore during the maintenance or setting adjustments. According to Sghaier, and Charpentier [6], collision between robot parts and human limbs is the most common accident in the industry since design of some safety components are inappropriate. Other accident types between human and machine is also frequent. For example, the operator could have his fingers or hand jammed in a machine like in conveyor, gears or between two manipulator arm links. The aims of safety standards are to prevent man access to the robot's workspace or to stop the robot when an unexpected event in the workspace is detected by external sensors $[7,8]$. However, those sensors are inadequate or inappropriate within a hybrid workspace. In the future, the progressive rapprochement between human and machine during manipulative tasks will increase the accident risk [9]. In order to reduce this risk, an implementation of adaptive safety systems upgraded for already installed industrial robot manipulator in a flexible manufacturing system is the main contribution of this paper.

The paper is organized as follows: in section II a literature review about safety in hybrid workspace and contributions of the paper are presented. In section III, a new safety system using an instrumented safety helmet and RSSIbased collision avoidance is defined. Section IV is devoted to the human localization in order to gives insight about 
possibility of collision without usage of camera. Finally, experimental results are reported in section $\mathrm{V}$ and the conclusion in section VI.

\section{RELATED WORK}

In flexible manufacturing system, it is only recently that human safety aspects and research on a safe interaction have become primordial. Therefore, the main motivation of this research project is to reduce the risk from possible injury caused by an industrial robot manipulator.

We can differentiate three categories related to the safety improvements in human-robot interactions [10]. The first one is the ability of robot to detect and to avoid collisions. However, if the contact is detected too late or if a problem occurs with the sensors or controllers, it must be ensured that no serious injury will be caused by the robot $[11,12]$. The second category is related to the operator's mental state. Indeed, lack of concentration, stress and fatigue, increase the risk of accident. Finally, the last category is related to environmental factors such as extreme temperature and lighting conditions that can affect the robot's sensors. In this paper, research work is dedicated on the first category which is based on collision avoidance system.

This section presents a review about obstacle detection using camera-based system and some approach employed in collision avoidance. Collision and physical impact with robot are also discussed and then the contribution of this paper is presented.

\section{A. Camera-based motion traking}

Many approaches on collision avoidance using camerabased system have been studied in the literature. This section discusses some different approaches.

Firstly, a reactive system of collision avoidance could be suggested. Before any collision, an algorithm could compute an estimated trajectory of human limbs and then plans an avoidance path. Such algorithm, based on camera sensors and SKELETON algorithm, is presented in [13]. This technique uses the camera to track the head motion. Despite experiments completed in laboratory which have obtained remarkable results, some other situations have not been experimented yet, such as: interference between an obstacle and the camera visual field of view, or the situation of multiuser inside workspace.

Low-cost approach are presented in [14] which uses multiple Kinect (Microsoft Kinect) for tracking human limbs. Distance between human and robot is evaluated in a 3D space in order to avoid collisions. Finally, Cherubini and al. [15] use also the Kinect and one another camera rigidly linked to the robot's end effector for human intention recognition and human-robot collaboration in industry environment. The system is based on a multimodal control approach and a state machine.

In these approaches, safety is improved. However, they have some drawbacks for collision detection which is based on the human limbs tracking. Indeed, the use of cameras raises several important issues, such as the computation burden, lighting conditions and light reflections with metal or glass parts (installed on a car's door for example) that can affect image quality and then reduce the capacity of realtime limb detection and tracking.

\section{B. Collision avoidance algorithm}

Among the others, the Artificial Potential Field approach [16] is the most famous and feasible method for collisions avoidance. The idea comes from permanent magnet: obstacle generates a repulsive pressure and the target an attractive pressure on all the surface of the robot. Hence, the robot is attracted to the target while avoiding obstacles. The main drawback of this approach is the presence of local minima [17]. Wallhoff and al. [18] propose a hybrid assembly station where the manipulator movements are adapted with the virtual force approach in order to ensure the human's safety. The obstacles avoidance is based on dynamic 3D environment model using depth sensing cameras. However, it requests high sensors reliability and telecommunication robustness.

\section{Physical interaction and collision management}

Detection and collision avoidance are essential to ensure safety in human-robot interaction. However, in the event of an unexpected impact, for example when sensors data are wrong, the robot can cause serious injury to the operator. For overcoming this issue, the robot must be designed in order to be intrinsically safe, in other words, physically incapable of being dangerous. The design of this type of robot is an essential step in order to permit a safe interaction. A famous robot safe intrinsically is called LWR III (Light weight robot), presented in [19]. This robot, which is developed at German Aerospace Center, is characterized by low inertia, torque sensing in each joint and an excellent ratio between the payload and the total mass. Haddadin and al. [20] propose a crash-testing methodology. The values obtained have shown that impact between human and robot does not cause serious injury. Another approach is based on motor current (proprioceptive) sensors which is detailed in [21]. The method used enables to distinguish between trajectory following, accidental collision and expected collaboration with the human through high pass filter and low pass filter respectively on the measurement of motor currents. However, the limited bandwidth of each states of the manipulator could decrease trajectory following performance.

As sensors reliability could be an issue, a mechanism which dislocates an axis of the manipulator in the event of collision is suggested in [22]. This system contains torque limiter located at the nearest axis of the end-effector: if a collision occurs and the torque limiter is exceeded, the manipulator becomes passive and the operator is only subjected to the inertia of the end-effector and the force related to the torque threshold. Another approach, rarely used, is to endow the robot with a sensory layer (a kind of robotic skin) to detect collisions [23] and manage interaction. 


\section{Contributions}

Our research project is focused on safety improvement in a hybrid workspace. In order to address this issue, the robot, during the handling, should detect human intentions in order to differentiate collaborative task and abnormal activities. In contrast with video-based detection devices, an Inertial Measurement Unit (IMU) using RSSI, such as used in [24], are chosen for detection and recognition of human activities in hybrid workspace because they are lighter and cheapest. The research work in this paper proposes human activities differentiation and trajectory avoidance of the operator in real time, based on an instrumented safety helmet. Human's position is estimated and the human activities are recognized, through the combination of IMU sensor and RSSI that are mounted into the instrumented safety helmet. Collisions avoidance methods that use the artificial potential field are employed on an industrial robot in order to produce a safe path.

\section{PROPOSED SYSTEM}

The system contains an instrumented safety helmet which recognizes the head gestures and localizes the users during their occupation. Furthermore, in order to complete the experiments in an industrial environment a flexible manufacturing system is also proposed. A block diagram of the operator's detection and collision avoidance algorithm are presented in Fig. 1. The data recovered from the instrumented safety helmet allow localization and activities recognition of the users. Then data are sent by wireless transmission to OPC server and robot's controller in order to produce a safe path planning.
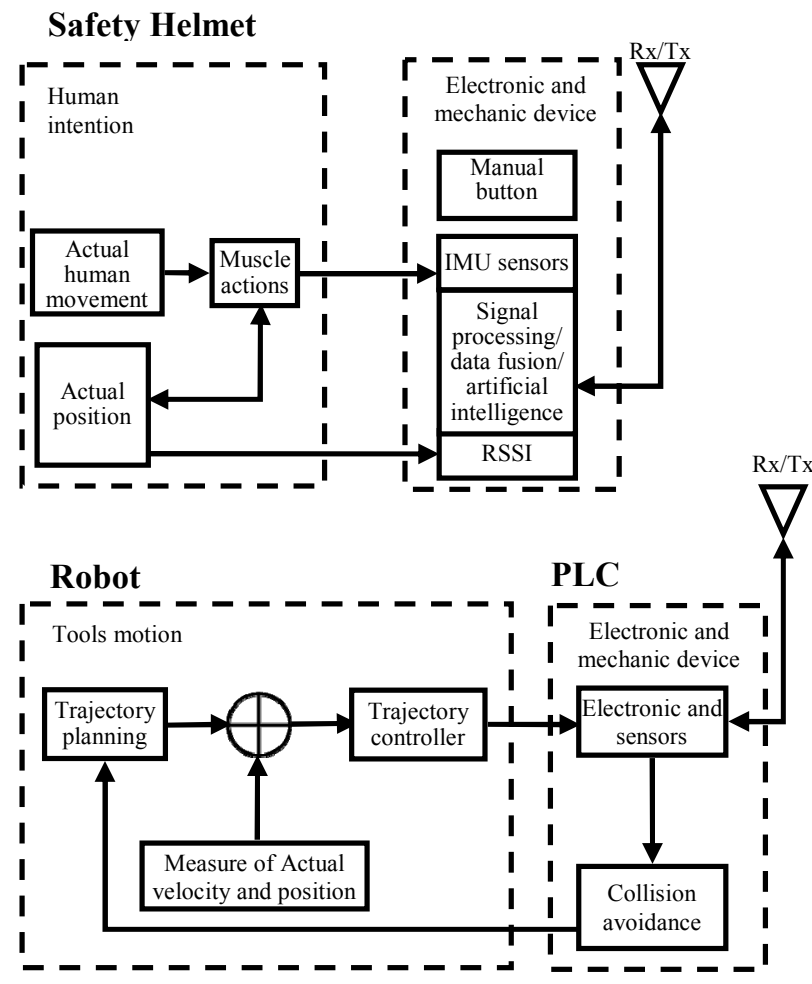

Figure 1: Block diagram of the interactive system

\section{A. Instrumented Safety Helmet}

The Safety Helmet system, as shown in Fig. 2, is an inexpensive, non-intrusive, non-invasive, and non-visionbased system, which consists in an Inertial Measurement Unit (IMU) module and localization system using WiFi signal strength labelled RSSI. RSSI gives an information about the operator position as suggested in [25]. IMU sensor is a device able to measure the moving object's acceleration, velocity, and orientation, employing a combination of three sensors using three axis measurements: 3DOF accelerometers, 3DOF gyroscopes and 3DOF magnetometers. Both IMU and RSSI signals acquired are processed by a Kalman filter which is implemented in a PIC24 microcontroller in order to evaluate localization and human activities recognition. Electronic board located inside the helmet, is the core unit of the artificial intelligence module. A haptic device, such as vibrotactile motor, is integrated to the helmet in order to alert the operator when a risk is evaluated.

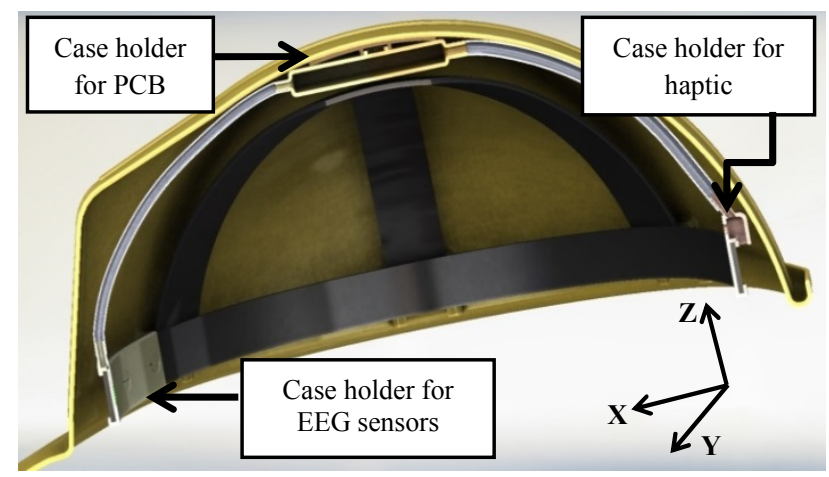

Figure 2: Sectional drawing of the Helmet [26]

\section{B. Flexible manufacturing system (FMS)}

Flexible manufacturing system (FMS), including a Programmable Logic Controller (PLC) and a robot, is shown in Fig. 3. The helmet communicates with the robot drive using an OPC server and a PLC. The information transmitted via OPC server are head position and activities of the operator. In this workspace, we can find other moving components such as a conveyor, a distributor and a storage system.

An automated assembly task is implemented in the FMS in order to allow both working time sharing and interactions. The task suggested for the evaluation of the safety helmet consists in assembling two different pieces, one made of plastic and the other made of steel. The assembly task proceeds as following: the operator cooperates with the robot by filling the distributors with the assembly pieces. The distributor pushes the first piece "A" on the conveyor then capacitive and optical sensors will determine the material that composes the piece "A". After this operation, the robot will grab the second piece, "B" on the second distributor, made of the same material than the first one "A". Before sending them in the storage system, the assembled pieces are checked with two other capacitive 
and optical sensors. Then, depending on the material with which pieces are made, the storage box is positioned thanks to a pneumatic cylinder. There are three positions for the different assembled pieces, steel-steel, plastic-plastic and steel-plastic (which is an assembly error). Operator has to manage assembly errors by the recuperation of the pieces.

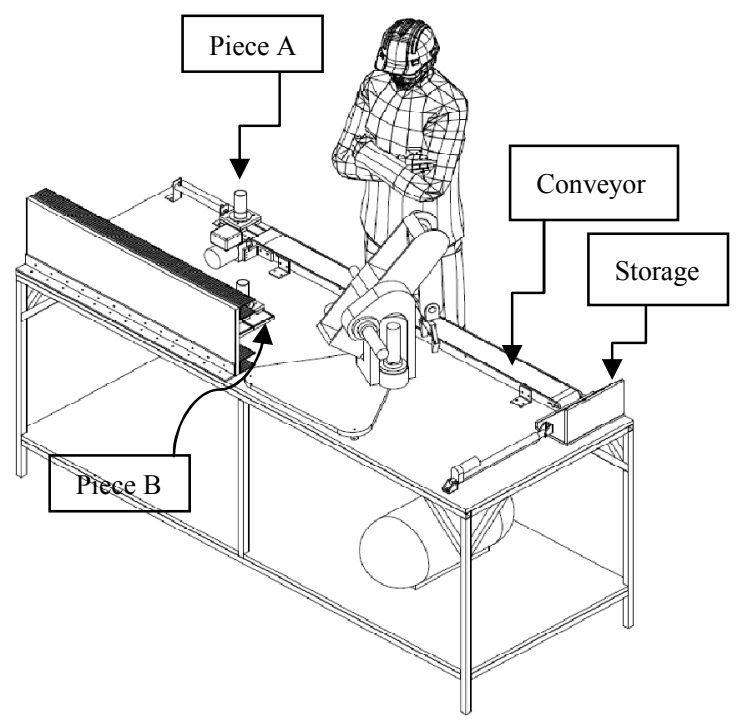

Figure 3: 3D isometric view of the FMS for the hybrid workspace

\section{METHODOLOGY}

In order to guarantee the operator's safety, the robot has to know the human's position and then it will be possible to plan a safe path thanks to a collisions avoidance approach. The experimental methodology is composed in two steps. Firstly, using an instrumented safety helmet in order to recognize head gestures and localize the operator's position. Secondly, developing an avoidance method, that produces a safe trajectory. In this research work, an ethics certification was obtained in order to practice the experiments on hybrid workspace.

The next subsections describe the algorithm and approach used in order to avoid collision in a hybrid workspace.

\section{A. Operator relative position from the end-effector}

The proposed technique combines the inertial measurement obtained from the IMU sensor and the RSSI based position estimation algorithm. These sensors are placed in the safety helmet, therefore on the operator. The data recovered through the IMU is the magnetometer and accelerometer signals in order to recognize the human activities. The operator's position is localized using three fixed wireless instruments. Then, the head position is estimated by measuring RSSI using the trilateration approach. In order to improve position accuracy, resulting value is then processed with IMU using Kalman filter.

Hence, a database of gestures is required in order to make a clear differentiation of human activities inside the workspace. For working time sharing task, a dictionary is defined which includes three basic motions: filling distributor, escape robot trajectory (dodge) and bend down (get new boxes containing assembly pieces). Dodge activity is defined in order to evaluate collision avoidance algorithm. Other human activities and head gestures have been investigated in previous work [26].

\section{B. Collision avoidance algorithm}

The Artificial Potential Field (AFP) is introduced by Khatib in 1985 [27]. The idea is to create an artificial potential field $\left(U_{a r t}\right)$ in robot's environment. A minimal distance between the robot and the obstacle should be kept: when the distance is too short, the robot is repulsed by the obstacle because the potential is strong; however it is attracted by the target because the potential field is weak. The artificial potential field is a sum of both attractive and repulsive potential fields which is determined by (1) where $q$ is the robot's geometric configuration:

$$
U_{\text {art }}(q)=U_{a t t}(q)+U_{r e p}(q)
$$

The robot moves following the sum of attractive and repulsive forces generated by the attractive potential and the repulsive potential respectively. The artificial force is determined by (2):

$$
\mathrm{F}_{\mathrm{art}}(q)=\mathrm{F}_{\mathrm{att}}(q)+\mathrm{F}_{\mathrm{rep}}(q)
$$

where $F_{\text {att }}(q)$ the attractive force and $F_{r e p}(q)$ is the repulsive force at the current position $q$ of the robot. The attractive force is provided by the equation (3):

$$
\overrightarrow{F_{a t t}}=-\varepsilon\left[q-q_{t}\right]
$$

where $\varepsilon$ is a positive gain (similar to Hooke's law spring constant), $q$ is current position of the robot and $q_{t}$ is target's position. The repulsive force is provided by the equation (4):

$$
\left\{\begin{array}{ll}
\overrightarrow{F_{\text {rep }}}=\frac{\eta}{d^{2}}\left[\frac{1}{d}-\frac{1}{d_{0}}\right] \vec{\nabla} d & \text { if } d \leq d_{0} \\
0 & \text { if } d>d_{0}
\end{array},\right.
$$

where $d$ is the distance between the minimal robotobstacle, $d_{0}$ is the influence distance of the obstacle and $\eta$ is a positive gain (similar to Hooke's law spring constant).

\section{EXPERIMENTAL RESULTS}

Participants perform the activities around the robot in order to complete the dictionary of human activities. A simple explanation of the activity vocabulary was given to these participants in order to understand the motions and their sequences. There was no special training on the vocabulary execution. Four male students wear the instrumented safety helmet, and then they practice a working time sharing task in the flexible manufacturing system. Indeed, participants assist the robot in an assembly task by managing assembly errors and filling distributors. The IMU signal and the operator's position are acquired for the overall assembly process on a Samsung tablet using 
Android OS. These measurements are collected at a sampling frequency around $50 \mathrm{~Hz}$ which is enough for head motion tracking and human activities recognition.

Each assembly process was repeated three times per participant for a total of twelve acquisitions per activity that are used in the analysis. The experiment process is explained in section III.B. and may be summarized as following: the participant fills the distributors, then the distributor pushes the first piece on the conveyor. Then the robot assembles pieces on the conveyor. Afterward, the user dodges robot arm. Finally, the participant leans in order to take the pieces in a new box situated on a second conveyor (bend down).

(a) Filling distributors
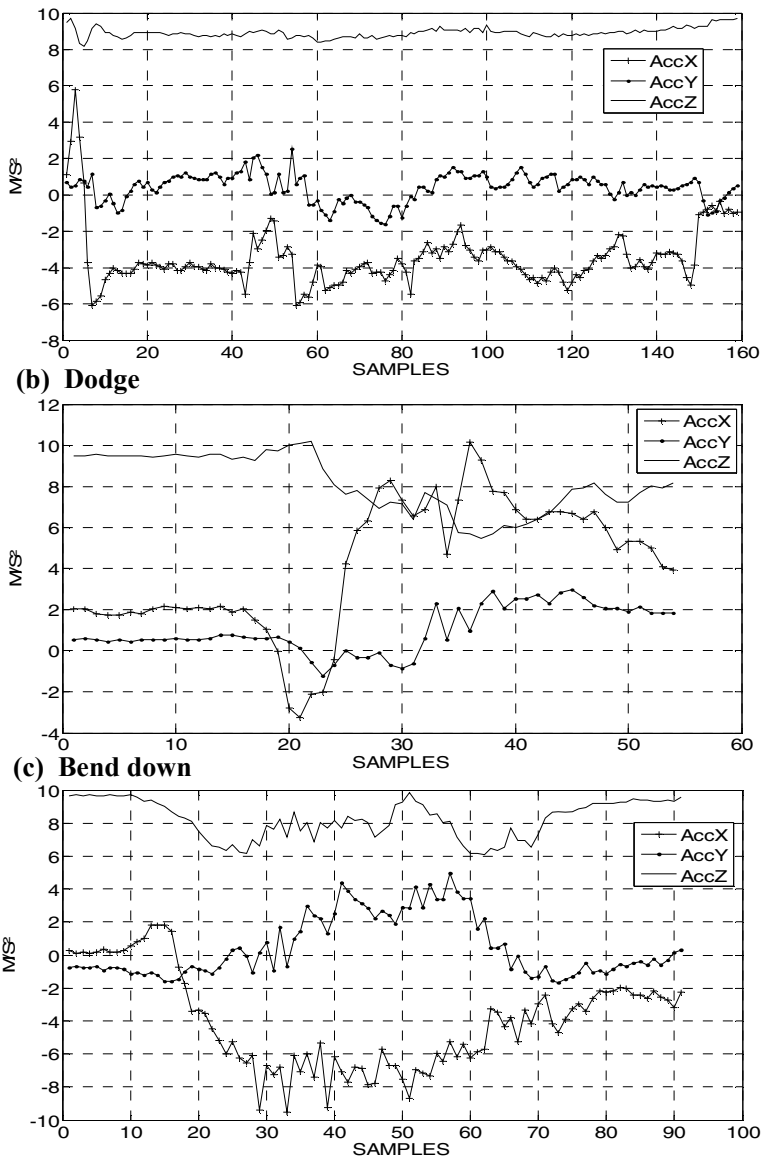

Figure 4: Acceleration signals of each human activity

\section{A. Results and analysis}

A statistical process is applied on all data by computing mean, variance, standard deviation and signal energy. This process allows to quantitatively differentiating human activities. It could also represent a first solution based on a features extraction process in time-domain for artificial intelligence algorithm. Fig. 4 shows the acceleration signals of each axis (X-axis, $\mathrm{Y}$-axis and $\mathrm{Z}$-axis) for each human activity coming from the first participant. Fig. 4(b) shows the acceleration waveform of dodge, which could be clearly differentiated from others signals shown in Fig. 4(a) and (c).

In order to differentiate activities, an analysis of variance (one way ANOVA) is used on the X-axis and Z- axis of the accelerometers. However, before applying ANOVA, mean, variance, standard deviation and energy are calculated of each activity in order to find an appropriate feature. These results were extracted for each participant, and then combined into a group. A total of twelve datasets of each DOF and each motion was saved into database for comparison purpose.

(a) Accelerometers axis $\mathrm{X}$

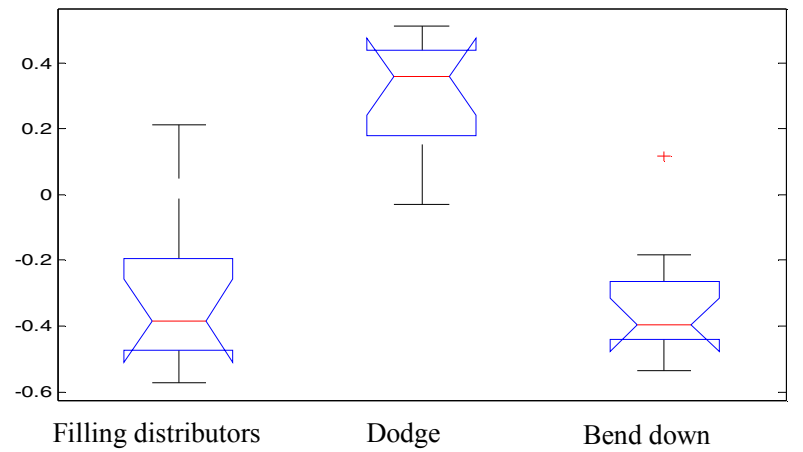

(b) Accelerometers axis $\mathrm{Z}$

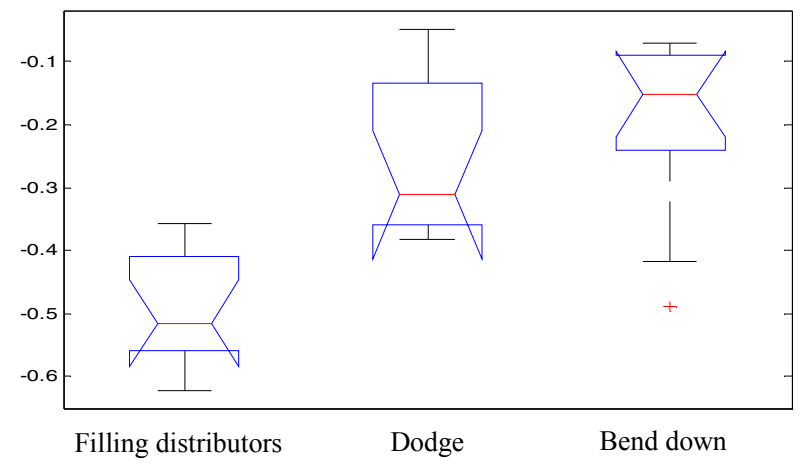

Figure 5: One-Way ANOVA on normalized acceleration

The normalized acceleration variance of $\mathrm{X}$-axis and $\mathrm{Z}$ axis are shown in Fig. 5(a) and Fig. 5(b) respectively. The p-value of $\mathrm{X}$-axis is $2.89 \times 10^{-10}$ which indicates that $\mathrm{X}$ axis could distinguish the dodge from bend down and filling distributor. The $\mathrm{p}$-value of $\mathrm{Z}$-axis is $8.71 \times 10^{-7}$ which indicate that acceleration of $\mathrm{Z}$-axis could discriminate bend down, dodge and filling activities. In conclusion, the acceleration variance of $\mathrm{X}$-axis could be used to identify dodge from human activities, while the acceleration variance of $Z$-axis could be used to identify bend down.

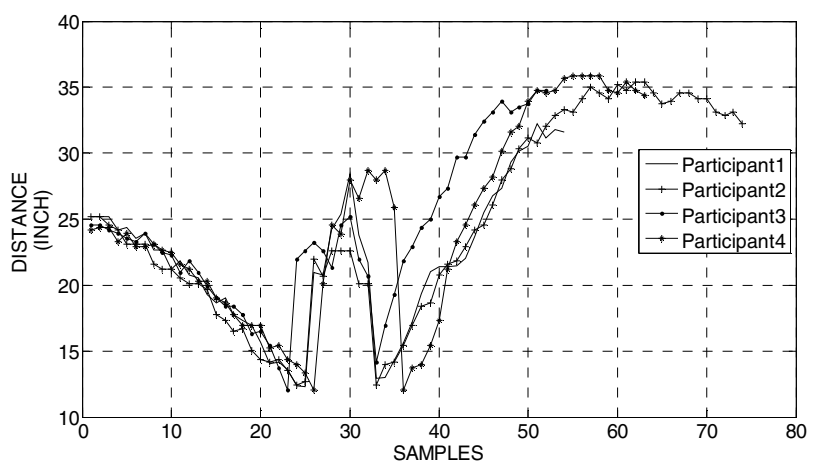

Figure 6: Distance between human and robot 
Finally, collision avoidance between robot and operator is shown in Fig. 6. Dodge activity was detected and then collision avoidance, using (4), was used with $\varepsilon=0.2$ and $\eta=-0.3$. The dashed line represents an estimate of the trajectory without collision avoidance. Of course, for the ethics certificate, collision is not allow any time, meaning a minimal distance of 5 inches is used. After a distance threshold $d_{0}=12$ inches, AFP is applied in order to generate a new trajectory for the robot. When the estimated robot trajectory reaches a minimal distance, it integrates the desired one in order to complete the assembly task as expected.

\section{CONCLUSION}

In this paper, a new interactive system is suggested for improving safety inside a flexible manufacturing system. Interaction with the robot can be complex while doing task such as assembly. In order to solve this issue, an operator's avoidance algorithm using an instrumented safety helmet implemented for hybrid workspace was presented. With the realized experiment, the combination of the IMU sensor and RSSI allows recognition of human activities and localization of the operator's position in real time. The Xaxis and Z-axis of the accelerometer could be adopted as indexes to differentiate each motion from others. A flexible manufacturing system was presented in order to simulate an industrial environment and a hybrid workspace.

In future work, the system should evaluate abnormal behavior which may lead to a collision or intentional physical contact. These situations could improve interactivity and then usability of the proposed flexible manufacturing system. Also, a risk of accident will be evaluated for monitoring operator and FMS.

\section{REFERENCES}

[1] J. E. Colgate, M. Peshkin, and H. S. Klostermeyer, "Intelligent Assist Devices in Industrial Applications: A Review," in IEEE/RSJ International Conference on Intelligent Robots and Systems, Las Vegas, Nevada, 2003, pp. $2516-2521$

[2] V. Duchaine, and C. Gosselin, "Passive Contact Reaction for Enhancing Human-Robot Collaboration," in ACM/IEEE International Conference on Human-Robot Interaction, Tokyo, Japon, 2013.

[3] J. Krüger, T. K. Lien, and A. Verl, "Cooperation of human and machines in assembly lines," CIRP Annals - Manufacturing Technology, vol. 58, no. 2, pp. 628-646, 2009.

[4] M. Zinn, O. Khatib, B. Roth, and J. K. Salisbury, "Playing It Safe," IEEE Robotics \& Automation Magazine, vol. 11, no. 2, pp. 12 - 21, June, 2004.

[5] A. Bicchi, M. A. Peshkin, and J. E. Colgate, "Safety for Physical Human-Robot interaction," Springer Handbook of Robotics, Springer-Verlag, ed., pp. 1335-1346, 2008.

[6] A. Sghaier, and P. Charpentier, "La problématique de l'utilisation des robots industriels en matière de sécurité," Annales des Mines Réalités industrielles, pp. 24-31, February 1, 2012.

[7] Association Française de Normalisation, "NF EN ISO 10218-1: Norme européenne : Norme française : Robots pour environnements industriels," AFNOR, 2006.

[8] Canadian Standards Association, "CAN/CSA-Z434-03: Robots industriels et systèmes robotiques exigences générales de sécurité," CSA, 2008.

[9] T. Malm, J. Viitaniemi, J. Latokartano, S. Lind, O. Venho-Ahonen, and J. Schabel, "Safety of Interactive Robotics-Learning from
Accidents," International Journal of Social Robotics, vol. 2, no. 3, pp. 221-227, 2010.

[10] M. Vasic, and A. Billard, "Safety Issues in Human-Robot Interactions," in IEEE International Conference on Robotics and Automation (ICRA) Karlsruhe - Germany, 2013, pp. 197 - 204.

[11] J. Heinzmann, and A. Zelinsky, "Quantitative Safety Guarantees for Physical Human-Robot Interaction," The International Journal of Robotics Research vol. 22, no. 7/8, pp. 479-504, 2003.

[12] N. Lauzier, and C. m. Gosselin, "Performance Indices for Collaborative Serial Robots With Optimally Adjusted Series Clutch Actuators," Journal of Mechanisms and Robotics, vol. 4, no. 2, pp. 021002, 2012.

[13] A. De Santis, and B. Siciliano, "Reactive collision avoidance for safer human-robot interaction," IARP-IEEE/RAS-EURON Workshop on Technical Challenges for Dependable Robots in Human Environments, Roma, Italy April, 2007.

[14] C. Morato, K. N. Kaipa, B. Zhao, and S. K. Gupta, "Toward Safe Human Robot Collaboration by Using Multiple Kinects Based RealTime Human Tracking," Journal of Computing and Information Science in Engineering, vol. 14, no. 1, pp. 011006-011006, 2014.

[15] A. Cherubini, R. Passama, A. Meline, A. Crosnier, and P. Fraisse, "Multimodal control for human-robot cooperation," in IEEE/RSJ International Conference on Intelligent Robots and Systems (IROS), Tokyo - Japan, 2013, pp. 2202 - 2207.

[16] A. Csiszar, M. Drust, T. Dietz, A. Verl, and C. Brisan, "Dynamic and Interactive Path Planning and Collision Avoidance for an Industrial Robot Using Artificial Potential Field Based Method," Mechatronics, pp. 413-421: Springer Berlin Heidelberg, 2012.

[17] F. Flacco, T. Kroger, A. De Luca, and O. Khatib, "A depth space approach to human-robot collision avoidance," in IEEE International Conference on Robotics and Automation (ICRA), RiverCentre, Saint Paul, Minnesota - USA, 2012, pp. 338 - 345.

[18] F. Wallhoff, J. Blume, A. Bannat, W. Rösel, C. Lenz, and A. Knoll, "A skill-based approach towards hybrid assembly," Advanced Engineering Informatics, vol. 24, no. 3, pp. 329-339, 2010.

[19] A. Albu-Schäffer, S. Haddadin, C. Ott, A. Stemmer, T. Wimböck, and G. Hirzinger, "The DLR lightweight robot: design and control concepts for robots in human environments," Industrial Robot: An International Journal, vol. 34, no. 5, pp. 376-385, 2007.

[20] S. Haddadin, A. Albu-Schäffer, and G. Hirzinger, "Safety Evaluation of Physical Human-Robot Interaction via Crash-Testing," in Robotics : Science and Systems Conference, Atlanta, United State, 2007, pp. 217-224.

[21] M. Geravand, F. Flacco, and A. De Luca, "Human-robot physical interaction and collaboration using an industrial robot with a closed control architecture," in IEEE International Conference on Robotics and Automation (ICRA), Karlsruhe, Germany, 2013, pp. 4000-4007.

[22] V. Duchaine, N. Lauzier, and C. Gosselin, "On the Design of HumanSafe Robot Manipulators," Robot Manipulators New Achievements, A. L. a. H. Kawai, ed.: InTech, 2010.

[23] V. Duchaine, N. Lauzier, M. Baril, M.-A. Lacasse, and C. Gosselin, "Flexible robot skin for safe physical human robot interaction," IEEE International Conference on Robotics and Automation (ICRA 2009), pp. 3676-3681, May, 12-17 2009.

[24] V. Garg, and M. Jhamb "A Review of Wireless Sensor Network on Localization Techniques," International Journal of Engineering Trends and Technology (IJETT), vol. 4, no. 4, April, 2013.

[25] W. Wong, L. S. Liew, C. H. Lai, and L. Liu, "Accurate Indoor Positioning Technique Using RSSI Assisted Inertial Measurement," Future Information Communication Technology and Applications, pp. 121-129, 2013.

[26] P. Li, R. Meziane, M. J.-D Otis, H. Ezzaidi, and P. Cardou, "A Smart Safety Helmet using IMU and EEG sensors for worker fatigue detection," in IEEE International Symposium on RObotic and SEnsors Environments (ROSE), Timisoara - Romania, Accepted, 2014.

[27] O. Khatib, "Real-time obstacle avoidance for manipulators and mobile robots," in IEEE International Conference on Robotics and Automation, 1985, pp. 500 - 505. 